\title{
电厂电气自动化系统改造与升级的探讨
}

俞浩

安徽华电宿州发电有限公司

DOI:10.32629/hwr.v4i4.2913

[摘 要] 现阶段,电气自动化技术被广泛应用在电厂建设中,为电厂运营及管理工作提供了保障。不过在电气自动化技术应用中,因受到传统技 术的影响, 使得自动化系统的功能得不到充分发挥,这对于电厂的运营及电能输送带来了极为不利的影响。为此, 电厂有必要对电气自动化系统 进行升级改造,不断创新和优化现有技术, 以此促进电力设备的安全稳定运行,降低人力、物力的损耗,提升总体经济效益。

[关键词]电厂; 电气自动化系统; 改造与升级

电气自动化系统的高效运转, 可对电厂内部各电气设施的运行情况予 以实时监督和管控, 及时发现和处理存在的故障问题, 进而减少因设备故 障引发的电能传输问题, 降低电厂运营中的风险系数, 维护企业的经济效 益。不过现阶段电气自动化系统存在一些滞后性, 与现今实际需求存在分 歧。为此, 就需要展开升级改造工作, 提高自动化控制水平, 确保企业的稳 定、安全发展。

\section{1 电厂电气自动化系统的构成}

电气自动化做到了电厂内部所有电气设备的实时监控、实时检测和分 析以及科学保护的功能, 将传统电气设备半自动监测及监管的模式转变成 集散控制模式, 达到了一个系统监管多台电气设备的目标, 加大了电厂管 控力度, 避免了危险的发生。通过对电厂电气自动化系统的研究, 了解到电 气自动化系统主要有三部分构成:

一是间隔层。间隔层内的设备采用的是分层间隔方式, 在开关层中设 置测控及保护装置, 对整个层内的设备进行监督和管理, 确保设备的独立 性。分层间隔的目的是规避因连接引发的故障问题, 降低设备维护次数及 成本。

二是网络通讯层。该层内设置了网络交换装置、通讯装置及中继器装 置等设备, 目的是进行设备系统间信息的实时传输, 保证各项指令下发及 执行的效率。

三是站控层。由工程师站、监测站、工作站、远程控制站四部分构成, 站控层的作用是实行信息收集、整合、处理及发送,一般会利用开放式和 分布式两种模式对改层予以科学管控, 以此促进电气设备的科学稳定运行, 减少故障问题的产生。

\section{2 电厂电气自动化系统存在的问题}

2.1系统控制问题

堤防安全评价是堤防设计和加固的重要组成部分。由于路堤地基岩土 的复杂物理力学性质、破坏机理和水文水力条件, 使得路堤的运行充满了 不确定性。基于概率论和可靠性的风险分析方法可以考虑实际工程中的不 确定性和荷载效应、阻力效应等各种影响因素的随机变异性, 在安全评价 中具有重要意义。

堤防工程防洪评价分析是一个比较新的研究领域, 是路堤工程、安全 评价方法和概率风险分析方法的交叉应用课题。堤防防洪评价是一项非常 复杂和精细的工作, 防洪系统中的各种工程措施都有各自的防洪和使用方 法。这些应用方法的研究的基础上,作者用系统的工程措施规划和统一的 方式调节洪水, 和获得最大的目标在保护区防洪效益, 进行系统的优化计 算, 分析和获得堤工程的泄洪能力, 以确定堤防工程的防洪标准的系统。

[参考文献]
目前, 我国电厂在系统控制上, 仍在采用传统的开关控制, 通过隔离开 关设置及按钮操作来实现系统的启动和关闭, 不过开关在长时间反复拨弄 的过程中, 很容易出现设备零部件质量问题, 影响控制效果, 使系统运行出 现各种问题, 造成一定的成本支出。为此, 就需要对系统控制模式实施优化 处理, 提升系统运行质量, 减少损失和危险的发生。

\section{2 监视系统设置不合理}

在传统自动化系统中设置的监视系统, 部分电气设备的运行情况并不 在监控范围内, 这导致设备运转中发生故障问题, 很难在第一时间发现和 解决, 连带着影响了连接设备或相邻设备的运行效果, 降低了电厂电气 自动化管控水平, 阻碍了电厂安全运营。根据对以往资料数据的分析可 知, 虽然每个电厂自动化系统中监控系统的设计都有其自身特征, 但是 总体结构上仍在采用传统的中央信号光字牌模式, 这些电厂监视系统在 运营中均存在监控不到位的问题, 无法对电气设备的稳定运行提供有效 保障, 进而降低了生产效益。另外, 传统的监视系统需要人工操作, 造成 人力成本的浪费。

2.3 运维人员综合能力素质不高

电厂在开展电气自动化设备的维护中, 部分单位的技术人员较为年轻, 缺乏工作经验, 加之相关技术人员的配备不齐全, 多是依靠厂家的维修人 员实施故障处理的, 所以在设备出现故障联系维修人员, 到厂家指派维修 人员到场维护这一阶段中, 存在着较多的不确定因素, 如厂家维修人员正 在其他区域工作、路途较远等, 使得维护工作被搁置, 一些问题无法及时处 理, 影响了电气自动化设备的正常使用, 为电厂带来较大的经济损失。

\section{3 电厂电气自动化系统的改造措施}

3. 1做好系统软硬件的设计和更新

电厂电气自动化系统的升级改造, 首要工作先是开展系统软硬件的升

[1]李青云,张建民.长江堤防安全评价的理论方法和实现策略 [J].中 国工程科学,2005, 7 (6):4-13.

[2]介玉新,胡蹈,李青云,等.层次分析法在长江堤防安全评价系统中的 应用 [J].清华大学学报(自然科学版),2004,44(12):1634-1637.

[3] 雷鹏, 肖峰,张贵金. 基于AHP的堤防安全评价系统研究 [J].人民黄 河,2013,35( 2 ):108-110+113.

[4]田林钢,靳聪聪, 巴超.改进的模糊层次分析法在海堤工程安全评价 中的应用[J].武汉大学学报(工学版),2013,46( 3 ):317-320+327.

[5]殷丹,石凤君,赵淑杰.河道堤防工程安全综合性评估[J].水利科技 与经济, 2013,18( 1 ):1-3.

[6]刘纯义,陈诚, 孙小艺. 黄河下游堤防安全评价指标的系统研究 [J]. 人民黄河,2006,27( 8 ):11-12. 
级和改造工作, 其中软件内容包括管理软件、系统平台软件、监控软件等 内容, 只有加强软件的实时性, 才能保证信息数据收集和处理的有效性, 推 动电气自动化系统的正常运转。硬件的升级改造要求工作人员做好技术优 化及日常维护工作, 借助日常维护来促进电气自动化控制系统的正常运转, 减少故障问题产生, 增强系统运行的高效性和安全性。技术优化则要求电 厂工作人员做好先进技术的引进和落实, 尤其要注重现场总线技术的引进 和更新, 以提高电厂的自动化管理水平, 完善电气自动化系统的运行效率。 同时, 通过现场总线技术的引入, 还能够实现网络信息技术与工业技术的 融合, 在一定程度上节省资源, 加强系统的可靠性, 减少电厂的生产成本, 增大企业的经济效益。

3.2 做好维护人员培养, 增强其综合能力

电厂电气自动化系统的设备在长时间运行中容易出现各种故障问题, 如零部件磨损、部件老化等, 需要专业维护人员对其实施科学处理。不过 在上文曾提到过, 电厂电气自动化系统设备的维护工作大多是由厂家负责 的, 电厂自身在维护工作中的参与积极性不高, 这导致电气自动化系统设 备故障产生后, 不能在第一时间予以解决, 造成经济上的损耗。为此, 电厂 有必要加大对电气自动化系统设备维护的重视力度, 设置专门的维修养护 部门, 并招募和培训专业技术人员, 这样在故障产生后, 工作人员能够第一 时间到场检查, 找出故障问题并加以解决。同时通过专业维护人员的培养, 还能降低维护中资金成本的损耗, 提高电气自动化系统运行的安全性。在 维护人员培训上, 要对设备安装流程、经常存在的故障问题及故障点予以 详细讲解, 借助与厂家技术人员的沟通交流, 增加自身的经验, 保证维护工 作的有效落实。

3. 3增强监视系统设置的合理性

针对传统监视系统存在的监管不到位情况, 电厂应对监视系统实行改 造升级, 在系统内部加入数据采集及处理功能, 做好信息数据的实时采集、 整理、分析及处理, 并将处理后的信息及时传送到通信管理模块内, 由通信 管理模块将数据上传和进一步解析, 以此确保总站及时了解各电气设备的 运转情况, 掌握设备相关参数的变化信息, 并及时对存在的异常数据信息 予以发现和处理, 降低系统运转的危险系数。再者, 在信息传输环节中, 需
要配备相应的显示器设备及报警系统, 将信息数据准确、直观的展现在工 作人员眼前, 对异常信息实施及时的报警处理。此外, 要不定期对数据采集 与通讯功能予以完善, 这是整个系统的基础, 主要负责系统中设备之间的 通讯、采集现场设备信息等。

3. 4 硬件配置优化

硬件配置一般以工作站的设置为主, 大多数情况下都是在集控室内 完成的。具体由一个主站系统、两个工作站、两台前置机、一台工程师 站组成。在前站机系统设置中, 自动切换时间要控制在 2 秒内, 手动切换 时间控制在 5 秒内, 主机与人机会话切换时间控制在 3 秒内, 命令切换时 间控制在 20 秒内。另外, 在网控室内还需设置三号RTU一台, 实现数据采 集控制及管理。

\section{4 结语}

随着经济的发展, 电能需求量的增多, 我国对电厂建设规模及要求也 在不断增加。为保证电厂内电气设备运转的高效性、安全性, 就有必要加 大电气自动化系统的改造和升级力度, 对软硬件设施实行更新改造, 以此 提高电气自动化控制水平, 改善设备运转效率, 进而维护电厂经济效益, 满 足现今社会的发展需求。

\section{[参考文献]}

[1]杜瑞.电厂电气自动化系统改造与升级 [J]. 当代化工研 究,2019(11):104-105.

[2]林丽琴.电厂电气自动化系统改造与升级 [J]. 当代化工研 究,2019(13):201+204.

[3] 孟志毅.电厂电气自动化系统改造与升级的探讨 [J].电子制 作,2013(11):171.

[4]代晓林. 电网系统中电气自动化应用技术升级问题[J]. 电子世 界,2018(11):175-176.

\section{作者简介:}

俞浩(1987--), 男, 安徽巢湖人, 汉族, 大学本科, 初级工程师, 从事工 作：安徽华电宿州发电有限公司集控运行。 